PROCEEDINGS OF THE

AMERICAN MATHEMATICAL SOCIETY

Volume 135, Number 9, September 2007, Pages 2689-2697

S 0002-9939(07)08790-4

Article electronically published on February 9, 2007

\title{
ROW AND COLUMN FINITE MATRICES
}

\author{
PACE P. NIELSEN
}

(Communicated by Martin Lorenz)

\begin{abstract}
Consider the ring of all $\kappa \times \kappa$ column finite matrices over a ring $R$. We prove that each such matrix is conjugate to a row and column finite matrix if and only if $R$ is right Noetherian and $\kappa$ is countable. We then demonstrate that one can perform this conjugation on countably many matrices simultaneously. Some applications and limitations are given.
\end{abstract}

\section{INTRODUCTION}

One learns in first year linear algebra that any linear transformation from one finite-dimensional vector space to another can be represented by a matrix of the appropriate size. In abstract algebra one shows that if $R$ is an associative ring with 1 , then an $R$-module homomorphism between free, right $R$-modules is similarly representable by a matrix. In fact, letting $F_{R}=R^{(\kappa)}$ be the free, right $R$-module on $\kappa$ generators, and thinking of the elements of $F_{R}$ as column vectors with $\kappa$ coordinates but with only finitely many of the coordinates nonzero, then one can identify $\operatorname{End}\left(F_{R}\right)$ with the ring, $\mathrm{CFM}_{\kappa}(R)$, of $\kappa \times \kappa$ matrices where each column has only finitely many nonzero entries. The ring $\operatorname{CFM}_{\kappa}(R)$ is called the ring of column finite matrices. We let $\operatorname{RCFM}_{\kappa}(R)$ be the subring of $\mathrm{CFM}_{\kappa}(R)$ consisting of matrices which are both row and column finite. We will focus on the case when $\kappa=\mathbb{Z}_{+}$and drop the subscripts in this case.

The ring $\operatorname{RCFM}(R)$ has begun to enjoy popularity in various settings. Recently it was shown that any countably generated algebra over a field $K$ can be embedded in $\operatorname{RCFM}(K)$ [3, Proposition 2.1]. This result, in turn, was used to define a new dimension function on such algebras [5]. The ring $\operatorname{RCFM}(R)$ has arisen in the context of Morita equivalence [4, exchange rings [1, 7, and Baer rings [2. There are still unanswered questions about this ring, such as whether or not $\operatorname{RCFM}(R)$ is clean (every element is the sum of an idempotent and a unit) even when $R$ is a field [6, Question 1].

Victor Camillo has raised the question of whether or not every element of $\operatorname{CFM}(R)$ is conjugate to an element in $\operatorname{RCFM}(R)$, when $R$ is a field. We answer his question in the positive and even classify all rings $R$ with this property. In the process we see that we can do this conjugation procedure to countably many matrices simultaneously.

Received by the editors November 19, 2005 and, in revised form, May 12, 2006.

2000 Mathematics Subject Classification. Primary 16S50; Secondary 16P40.

Key words and phrases. Row and column finite matrices, endomorphism ring, vector space. 
In the following, all rings will be associative with 1 . All modules will be unital. By $\mathbb{N}$ we will mean the nonnegative integers, and $\mathbb{Z}_{+}$is the set of positive integers.

\section{Conjugating matrices}

As a matter of notation for this section we fix $F_{R}=R^{\left(\mathbb{Z}_{+}\right)}$. If $T \in \operatorname{End}\left(F_{R}\right)$ and $B$ is a basis of $F_{R}$ we write $T_{B}$ to mean the $\mathbb{Z}_{+} \times \mathbb{Z}_{+}$column finite matrix representing the action of $T$ on $F_{R}$, in the basis $B$. We also write $\operatorname{supp}_{B}(x)$ to mean the support of a vector $x \in F_{R}$ when written in the basis $B$.

The first question one may ask is: What would stand in the way of a column finite matrix being conjugate to a row and column finite one? The easiest situation to try is a matrix with the top row infinite, all the other rows zero, and where the top row is badly behaved. This leads us to our first example.

Example 1. Let $R$ be a ring that is not right Noetherian. We have a chain of right ideals, $I_{1} \varsubsetneqq I_{2} \varsubsetneqq \ldots$ of $R$. For each $i \in \mathbb{Z}_{+}$fix $a_{i} \in I_{i} \backslash I_{i-1}$. This yields the chain

$$
a_{1} R \varsubsetneqq a_{1} R+a_{2} R \varsubsetneqq a_{1} R+a_{2} R+a_{3} R \varsubsetneqq \ldots
$$

of right ideals. Let $C=\left\{v_{1}, v_{2}, \ldots\right\}$ be a fixed basis for $F_{R}$. Let $T$ be the transformation defined by $T\left(v_{i}\right)=v_{1} a_{i}$, for each $i \in \mathbb{Z}_{+}$. Clearly, as it stands, $T_{C}$ is not row finite. (The matrix $T_{C}$ is the matrix with the $a_{i}$ along the top row, and zeros elsewhere.) Note that $\operatorname{im}(T)=v_{1}\left(a_{1} R+a_{2} R+\cdots\right)$.

Suppose, by way of a contradiction, that there is a basis $B=\left\{w_{1}, w_{2}, \ldots\right\}$ for $F_{R}$ under which $T_{B}$ is a row and column finite matrix. First, we can write $v_{1}$ as a finite linear combination in the $w_{j}$, say

$$
v_{1}=\sum_{j=1}^{n} w_{j} r_{j}
$$

for some $n \in \mathbb{Z}_{+}$, and $r_{j} \in R$ for each $j \leqslant n$. In particular,

$$
\operatorname{im}(T) \subseteq v_{1} R \subseteq \bigoplus_{j=1}^{n} w_{j} R .
$$

Since $T_{B}$ is row finite, the elements $\left\{w_{1}, \ldots, w_{n}\right\}$ can only appear in finitely many of the supports, $\operatorname{supp}_{B}\left(T\left(w_{j}\right)\right)$, for $j \in \mathbb{Z}_{+}$. Making $n$ large enough, we may then suppose $T\left(w_{j}\right)=0$ for $j>n$.

There is some $m \in \mathbb{Z}_{+}$so that $\left\{w_{1}, \ldots, w_{n}\right\}$ can all be written as linear combinations in the set $\left\{v_{1}, \ldots, v_{m}\right\}$. In particular, for $j \leqslant n$ we have

$$
T\left(w_{j}\right) \in T\left(v_{1}\right) R+\cdots+T\left(v_{m}\right) R=v_{1}\left(a_{1} R+\cdots+a_{m} R\right),
$$

and hence

$$
\operatorname{im}(T) \subseteq v_{1}\left(a_{1} R+\cdots+a_{m} R\right) \varsubsetneqq v_{1}\left(a_{1} R+a_{2} R+\cdots\right),
$$

yielding a contradiction. Therefore, $T$ cannot be written as a row and column finite matrix.

It turns out that this sort of situation is as bad as it gets, and so we obtain the following nice characterization:

Theorem 2. If $R$ is a ring, then $R$ is right Noetherian if and only if each matrix in $\operatorname{CFM}(R)$ is conjugate to a matrix in $\operatorname{RCFM}(R)$. 
Proof. In view of Example 1 we only need prove the "only if" direction. Let $T$ be an endomorphism of $F_{R}$. Given any basis $B$ of $F_{R}$, we write $T_{B,(i, j)}$ for the $(i, j)$-entry of $T_{B}$. Let $B_{1}=\left\{v_{1,1}, v_{2,1}, v_{3,1}, \ldots\right\}$ be a fixed basis for $F_{R}$.

For each $k \in \mathbb{Z}_{+}$we will construct a basis $B_{k}=\left\{v_{1, k}, v_{2, k}, \ldots\right\}$ satisfying the following five conditions:

1. The first $k$ vectors of both $B_{k}$ and $B_{k+1}$ agree.

2. For any $n \in \mathbb{Z}_{+}$, the span of the first $n$ vectors from $B_{k}$ equals the span of the first $n$ vectors of $B_{k+1}$.

3. The first $k-1$ rows of both $T_{B_{k}}$ and $T_{B_{k+1}}$ agree.

4. When passing from $T_{B_{k}}$ to $T_{B_{k+1}}$, the first $k$ columns do not increase in length.

5. The first $k$ rows of $T_{B_{k+1}}$ are finite.

Before we prove the existence of such bases, let us look at some consequences of these conditions. First, let $B_{\Delta}=\left\{v_{1,1}, v_{2,2}, v_{3,3}, \ldots\right\}$ be the set of diagonal elements arising from these bases. Then condition (1) implies that for any $k \in \mathbb{N}$ we also have $B_{\Delta}=\left\{v_{1, k}, v_{2, k}, \ldots, v_{k, k}, v_{k+1, k+1}, v_{k+2, k+2}, \ldots\right\}$. So, in some sense, $B_{\Delta}=\lim _{n \rightarrow \infty} B_{n}$. Second, by condition (2) we see that $B_{\Delta}$ is actually a basis for $F_{R}$.

Next, suppose that the $k$ th column of $T_{B_{k}}$ has length no longer than $d \geqslant k$. Then condition (4) implies that the $k$ th column of each of the matrices $T_{B_{k}}, T_{B_{k+1}}, \ldots$ is of length $\leqslant d$. We then see that the $k$ th column of each of the matrices $T_{B_{d+1}}, T_{B_{d+2}}, \ldots$ agree, since by condition (3) the first $d$ rows agree. Also, since the first $d$ vectors of $B_{d}$ and $B_{\Delta}$ agree, we see that this column is also the $k$ th column of $T_{B_{\Delta}}$. So $T_{B_{\Delta}}$ is the matrix which is the limit of the matrices $T_{B_{1}}, T_{B_{2}}, \ldots$. Finally, by conditions (3) and (5) we see that the rows of $T_{B_{\Delta}}$ must be finite, and hence $B_{\Delta}$ is the needed basis.

Now we prove existence. Working by induction, we may suppose that

$$
B_{k}=\left\{v_{1, k}, v_{2, k}, v_{3, k}, \ldots\right\}
$$

has been constructed for some $k \geqslant 1$. By hypothesis, the first $k-1$ rows of $T_{B_{k}}$ are finite, so we set

$$
p_{k}=\max \left\{j \mid T_{B_{k},(i, j)} \neq 0 \text { for some } i \in[1, k-1]\right\} .
$$

In other words, $p_{k}$ is the maximum length of the first $k-1$ rows. (If $k=1$ we set $p_{k}=1$.) Set $m_{k}=\max \left\{k+1, p_{k}\right\}$, and let $J_{k}$ be the right ideal generated by the entries $T_{B_{k},(k, j)}$ for $j \geqslant m_{k}+1$. Since $R$ is right Noetherian, there is some integer $n_{k} \geqslant m_{k}+1$ such that $J_{k}$ is generated by $T_{B_{k},(k, j)}$ for $j \in\left[m_{k}+1, n_{k}\right]$.

Finally, we are ready to define $B_{k+1}=\left\{v_{1, k+1}, v_{2, k+1}, \ldots\right\}$. For $\ell \leqslant n_{k}$, we put $v_{\ell, k+1}:=v_{\ell, k}$. For $\ell>n_{k}$, we put

$$
v_{\ell, k+1}:=v_{\ell, k}+\sum_{i=m_{k}+1}^{n_{k}} v_{i, k} c_{i, \ell}
$$

for some $c_{i, \ell} \in R$, to be chosen shortly. In other words, the change of basis matrix is of the form

$$
U_{k}:=\left(\begin{array}{ccc}
I_{m_{k}} & 0 & 0 \\
0 & I_{n_{k}-m_{k}} & C \\
0 & 0 & I_{\mathbb{Z}_{+}}
\end{array}\right),
$$


where $C$ is the matrix formed from the (unspecified) constants $c_{i, \ell}$, and $I_{\bullet}$ is the $\bullet \times$ identity matrix. One checks directly that

$$
U_{k}^{-1}=\left(\begin{array}{ccc}
I_{m_{k}+1} & 0 & 0 \\
0 & I_{n_{k}-m_{k}} & -C \\
0 & 0 & I_{\mathbb{Z}_{+}}
\end{array}\right) .
$$

Because $T_{B_{k+1}}=U_{k}^{-1} T_{B_{k}} U_{k}$, we only need to show that after these matrix multiplications our five conditions are met.

Right multiplication by $U_{k}$ corresponds to column operations. So $T_{B_{k}} U_{k}$ is the matrix formed by taking $T_{B_{k}}$ and adding $c_{i, \ell}$ times the $i$ th column to the $\ell$ th column (for $i \in\left[m_{k}+1, n_{k}\right]$ and $\ell>n_{k}$ ). But because $J_{k}$ is generated by the entries along the $k$ th row in these columns, we can choose the $c_{i, \ell}$ so that the $k$ th row of $T_{B_{k}} U_{k}$ becomes 0 after the $n_{k}$ th column. (In other words, we "column reduce" along the $k$ th row, after a specified point.)

Also notice that the first $k-1$ rows of $T_{B_{k}}$ are not affected by right multiplication by $U_{k}$, because we chose $m_{k} \geqslant p_{k}$. Therefore, the first $k$ rows of $T_{B_{k}} U_{k}$ are finite. Also, because $m_{k}>k$, the first $k$ rows of $T_{B_{k}} U_{k}$ are unchanged by left multiplication by $U_{k}^{-1}$, and hence condition (5) holds. These facts then imply that the first $k-1$ rows of $T_{B_{k}}$ and $T_{B_{k+1}}$ agree, yielding condition (3). Conditions (1) and (2) are obvious from the construction. Finally, right multiplication by $U_{k}$ doesn't change the first $k$ columns, and left multiplication by $U_{k}^{-1}$ corresponds to adding rows upwards (since this matrix is upper-triangular) and so cannot increase column length. Hence condition (4) also holds.

The method of proof actually gives a construction of the conjugating matrix. It is $U:=\lim _{n \rightarrow \infty} U_{1} U_{2} \cdots U_{n}$, and hence we can actually accomplish this conjugation by an upper-triangular matrix with 1's down the diagonal.

Analyzing the proof one notices that what we really did was column reduce one row at a time, making sure that when we multiply by the inverse matrix on the left that we don't undo our work, and also making it so that the limit of the product of these invertible matrices exists and is still invertible.

Suppose now that we have a $\mathbb{Z}_{+} \times \kappa$ column finite matrix $M$ over a right Noetherian ring $R$, where $\kappa$ is arbitrary. Conjugation now makes no sense, but we can still ask whether there is an invertible, $\kappa \times \kappa$ column finite matrix $U$, for which $M U$ is row and column finite. It is not difficult to modify the above argument to show that such a matrix $U$ exists, as follows:

First, notice that a submodule $L$ of $F_{R}$ is always countably generated as long as $R$ is right Noetherian. This is because $L=\bigcup_{n \in \mathbb{Z}_{+}} L_{n}$ where $L_{n}=L \cap\left(e_{1} R+\cdots+e_{n} R\right)$, with $\left\{e_{1}, e_{2}, \ldots\right\}$ a basis for $F_{R}$. Each $L_{n}$ is finitely generated (being a submodule of $e_{1} R \oplus \cdots \oplus e_{n} R \cong R_{R}^{n}$, which is right Noetherian), so $L$ is countably generated. Therefore, the columns of $M$ generate a countably generated submodule of $F_{R}$. After multiplying $M$ on the right by a permutation, we may assume that the first $\mathbb{Z}_{+}$ columns of $M$ generate the column space. But then, using these first $\mathbb{Z}_{+}$columns, we can column reduce the remainder of the columns of $M$ to zero. Finally, we can do column reductions among these first $\mathbb{Z}_{+}$columns, to make $M$ row and column finite, just as in Theorem 2 .

Homologically, these last few paragraphs prove that every countably generated module over a right Noetherian ring has a "row and column finite, free resolution". What we mean by this is: 
Proposition 3. Let $M$ be a countably generated module over a right Noetherian ring. Let

$$
\cdots \stackrel{\varphi_{3}}{\longrightarrow} F_{2} \stackrel{\varphi_{2}}{\longrightarrow} F_{1} \stackrel{\varphi_{1}}{\longrightarrow} F_{0} \longrightarrow M \longrightarrow 0
$$

be a free resolution of $M$ with each $F_{n}$ countably generated. (Such a resolution is possible from our work above.) Then there exist bases $B_{n}$ of $F_{n}$, for each $n \in \mathbb{N}$, so that the maps $\varphi_{n+1}: F_{n+1} \rightarrow F_{n}$ are represented by row and column finite matrices in these bases. In other words, each element $b \in B_{n}$ occurs in the support of only finitely many elements of $\varphi_{n+1}\left(B_{n+1}\right)$.

\section{Simultaneous conjugation and Embeddings}

Let $T_{1}, \ldots, T_{r} \in \operatorname{CFM}(R)$, for some $r \in \mathbb{Z}_{+}$. One may wonder if we can modify the proof of Theorem 2 in such a way that $T_{1}, \ldots, T_{r}$ will simultaneously conjugate to row and column finite matrices. The answer is yes, and in fact the proof will work for countably many matrices $T_{1}, T_{2}, \ldots \in \operatorname{CFM}(R)$ all at once!

Theorem 4. Let $R$ be a right Noetherian ring. For each countable set of elements of $\operatorname{CFM}(R)$ there exists an invertible matrix in $\operatorname{CFM}(R)$ that conjugates the set into $\operatorname{RCFM}(R)$.

Proof. We again want to inductively construct a sequence of bases for $F_{R}=R^{\left(\mathbb{Z}_{+}\right)}$ so that the diagonal set is a basis with the right properties. The trick is that since there are only countably many elements $T_{1}, T_{2}, \ldots \in \operatorname{End}\left(F_{R}\right) \cong \operatorname{CFM}(R)$, there are also only countably many rows that we need to column reduce. Let $r_{m, n, B}$ be the $m$ th row of $T_{n, B}$, under a basis $B$ for $F$. Then we order all the rows $\left\{r_{m, n, B}\right\}$ by a diagonalization well-ordering: $r_{m, n, B} \leqslant r_{m^{\prime}, n^{\prime}, B}$ if and only if $m+n<m^{\prime}+n^{\prime}$, or $m+n=m^{\prime}+n^{\prime}$ and $m \leqslant m^{\prime}$. Whenever we speak of an ordering on $\left\{r_{m, n, B}\right\}$ we are talking about this well-ordering.

The proof goes as before, with the following small changes. First, conditions (3) through (5) in the inductive construction must be restated.

3. The first $k-1$ rows of both $\left\{r_{m, n, B_{k}}\right\}$ and $\left\{r_{m, n, B_{k+1}}\right\}$ agree.

4. When passing from $T_{n, B_{k}}$ to $T_{n, B_{k+1}}$, the first $k$ columns do not increase in length, for each $n \in \mathbb{Z}_{+}$.

5 . The first $k$ rows of the set $\left\{r_{m, n, B_{k+1}}\right\}$ are finite.

Second, we have to modify the definition of $p_{k}$. We now let $p_{k}$ be the maximum length of the first $k-1$ rows of the set $\left\{r_{m, n, B_{k}}\right\}$, which are finite by our inductive hypothesis. We again set $m_{k}=\max \left\{k+1, p_{k}\right\}$.

Third, let $r_{m^{\prime}, n^{\prime}, B_{k}}$ be the $k$ th row in the set $\left\{r_{m, n, B_{k}}\right\}$. We modify the definition of $J_{k}$ by letting it equal the module generated by the entries $T_{n^{\prime}, B_{k},\left(m^{\prime}, j\right)}$, for $j>$ $m_{k}$. Since $R_{R}$ is Noetherian, there is some number $n_{k}>m_{k}$ so that $J_{k}$ is generated by these entries for $j \in\left[m_{k}+1, n_{k}\right]$.

The conjugating matrices are defined exactly as before, and the constants are chosen so that we column reduce the row $r_{m^{\prime}, n^{\prime}, B_{k}}$. The rest of the proof is unchanged. The only other point that needs mentioning is the fact that if $r_{m^{\prime \prime}, n^{\prime \prime}, B_{k}}$ is the $i$ th row of the set $\left\{r_{m, n, B_{k}}\right\}$, for some $i \leqslant k$, then $m^{\prime \prime} \leqslant k$ (which follows from how our well-ordering is defined). This implies that left multiplication by $U_{k}^{-1}$ will not affect such a row.

Theorem 4allows us to conjugate sets of equations from $\operatorname{CFM}(R)$ into $\operatorname{RCFM}(R)$. 
Example 5. Let $R$ be a field. It is well known that $\operatorname{CFM}(R)$ is clean. That is, for any element $x \in \operatorname{CFM}(R)$ there exist $e, u, v \in \operatorname{CFM}(R)$ so that $x=u+e$, $e^{2}=e$, and $u v=v u=1$. We can conjugate these equations by some element $\sigma \in \mathrm{U}(\operatorname{CFM}(R))$ so that the resulting equations $x^{\sigma}=u^{\sigma}+e^{\sigma},\left(e^{2}\right)^{\sigma}=e^{\sigma}$, and $u^{\sigma} v^{\sigma}=v^{\sigma} u^{\sigma}=1^{\sigma}=1$ hold in $\operatorname{RCFM}(R)$. Thus $x^{\sigma}$ is clean, even in $\operatorname{RCFM}(R)$.

If we started with $x \in \operatorname{RCFM}(R) \varsubsetneqq \operatorname{CFM}(R)$ in the first place, one might wonder whether we can choose $\sigma$ so that $x^{\sigma}=x$, and this would show that $\operatorname{RCFM}(R)$ is clean. However, using the methods developed here, this is impossible without knowing more about $e, u$, and $v$.

Conversely, let $R$ be a right Noetherian ring for which $\operatorname{RCFM}(R)$ is clean. Given $x \in \operatorname{CFM}(R)$ we can find $\sigma \in \mathrm{U}(\operatorname{CFM}(R))$ so that $x^{\sigma} \in \operatorname{RCFM}(R)$. Then we can find $u \in \mathrm{U}(\operatorname{RCFM}(R))$ and $e^{2}=e \in \operatorname{RCFM}(R)$ so that $x^{\sigma}=u+e$. Then $x=u^{\sigma^{-1}}+e^{\sigma^{-1}}$, and hence $\operatorname{CFM}(R)$ is clean. Unfortunately, I know of no nonzero ring $R$ where it is proven that $\operatorname{RCFM}(R)$ is clean.

Since we can actually conjugate countably many elements simultaneously, we can do more than push equations into $\operatorname{RCFM}(R)$. For example, we can give a new (and slightly more general) proof of the following:

Corollary 6 ([3, Proposition 2.1]). Let $K$ be a field, and let $A$ be a countably generated $K$-algebra. Then $A$ can be embedded in $\operatorname{RCFM}(K)$.

Proof. Consider the $K$-vector space $A_{K}$. We can embed $A$ (as a $K$-algebra) into $\operatorname{End}\left(A_{K}\right) \cong \operatorname{CFM}(K)$ via the left regular representation. But then, by Theorem 4 we can conjugate generators for $A$ into $\operatorname{RCFM}(R)$.

Note that this proof only required $K$ to be commutative Noetherian and the countably generated $K$-algebra $A$ to be free as a $K$-module. Even more generally, we have:

Corollary 7. Let $R$ be a right Noetherian ring, and let $A$ be a countably generated right $R$-module. If $A$ embeds into $\operatorname{CFM}(R)$, then $A$ embeds into $\operatorname{RCFM}(R)$. Furthermore, if $A$ is a ring and the embedding of $A$ into $\operatorname{CFM}(R)$ is both a ring and a right $R$-module embedding, then so is the embedding into $\operatorname{RCFM}(R)$.

\section{Limitations AND RESTRICTIONS}

Theorem 4 is surprising. One might wonder whether uncountably many (or more specifically, $\aleph_{1}$ many) matrices in $\operatorname{CFM}(R)$ could be simultaneously conjugated into $\operatorname{RCFM}(R)$. The following example negates this possibility.

Example 8. Let $R$ be a nonzero ring. Let $U \in \mathrm{U}(\operatorname{CFM}(R))$ be an arbitrary, invertible, column finite matrix. Consider another matrix $M \in \operatorname{CFM}(R)$ whose only nonzero entries occur on the top row, and this row has infinitely many nonzero entries. Notice that the only nonzero entries of $M U$ also occur on the top row. If we write

$$
M U=\left(\begin{array}{cccc}
a_{1} & a_{2} & a_{3} & \ldots \\
0 & 0 & 0 & \ldots \\
0 & 0 & 0 & \ldots \\
\vdots & \vdots & \vdots & \ddots
\end{array}\right)
$$

and write $U^{-1}=\left(\begin{array}{llll}c_{1} & c_{2} & c_{3} & \cdots\end{array}\right)$ in terms of its columns, then

$$
U^{-1} M U=\left(\begin{array}{llll}
c_{1} a_{1} & c_{1} a_{2} & c_{1} a_{3} & \cdots
\end{array}\right) .
$$


If $c_{1} a_{i}=0$, then $a_{i}=0$, because if we let $r_{1}$ be the top row of $U$, then $r_{1} c_{1}=1$. Thus we see that if $M U$ isn't row finite, then neither is $U^{-1} M U$.

On the other hand, let $B=\left\{v_{1}, v_{2}, \ldots\right\}$ be the set of columns of $U$. Then $B$ is a basis for $F_{R}=R^{\left(\mathbb{Z}_{+}\right)}$. Let $x$ be the top row of $M$. We have that $M U$ is row finite if and only if there exists some $n \in \mathbb{Z}_{+}$so that $x v_{i}=0$ for $i>n$ (where by $x v_{i}$ we mean the matrix multiplication of a row by a column). In other words, $x$ is (in some sense) perpendicular to the $v_{i}$. But note that $x^{T} \notin F_{R}$ since $x$ has infinitely many nonzero entries.

Let $C=\left\{e_{1}, e_{2}, \ldots\right\}$ be the standard basis for $F_{R}$. For each $n \in \mathbb{Z}_{+}$let $m_{n} \in$ $\mathbb{Z}_{+}$be the smallest number so that $e_{j} \notin \operatorname{supp}_{C}\left(v_{i}\right)$ for all $i \leqslant n, j \geqslant m_{n}$. Let $y=\left(\begin{array}{lll}y_{1} & y_{2} & \cdots\end{array}\right)$ be a row vector, where the first $m_{n}$ entries are fixed, and such that $y v_{i}=0$ for all $i>n$. We claim that the other entries of $y$ are then uniquely determined. In fact, for any $i>m_{n}$ we have $e_{i}=z_{i}+z_{i}^{\prime}$, where $z_{i} \in \operatorname{Span}\left(v_{1}, \ldots, v_{n}\right)$ and $z_{i}^{\prime} \in \operatorname{Span}\left(v_{n+1}, v_{n+2}, \ldots\right)$. Then

$$
0=y z_{i}^{\prime}=y\left(e_{i}-z_{i}\right)=y_{i}-y z_{i}
$$

and so $y_{i}=y z_{i}$, which is uniquely determined by $U$ and the first $m_{n}$ entries of $y$.

Thus, there is at most one row that is perpendicular to each $v_{i}$, for $i>n$, once the first $m_{n}$ coordinates of that row have been fixed. Let $S$ be any set of rows of cardinality $\aleph_{1}$, where the entries are limited to 1's and 0's. (Such a set exists, since the set of all countable sequences in 1's and 0's has continuum cardinality.) Note that the definition of $S$ does not depend on $U$. Now for each $m \in \mathbb{Z}_{+}$there are only finitely many choices for the first $m$ coordinates of the vectors in $S$. Hence there are at most finitely many vectors in $S$ that are perpendicular to all but the first $n$ columns of $U$. In particular, there are at most countably many vectors in $S$ that are perpendicular to all but finitely many of the columns of $U$. Therefore, right multiplication by $U$ cannot simultaneously send all the elements of $S$ into finite rows, and hence if we put each element of $S$ as the first row of a matrix in $\operatorname{CFM}(R)$ (with the other rows set to zero), we see that these uncountably many matrices cannot be simultaneously conjugated into $\operatorname{RCFM}(R)$.

If one isn't worried about $\aleph_{1}$, there is an easier way to verify our claim. Let $R$ be a nonzero ring, let $U$ be an invertible matrix in $\operatorname{CFM}(R)$, and let $A$ be a nonzero right ideal in $\operatorname{CFM}(R)$. One easily checks that nonzero right ideals of $\operatorname{CFM}(R)$ cannot be contained in $\operatorname{RCFM}(R)$. But $U^{-1} A U$ is such a right ideal. Thus $A$ cannot be conjugated into $\operatorname{RCFM}(R)$.

Another question this work raises is whether or not elements in $\operatorname{CFM}_{\kappa}(R)$ can be conjugated into $\operatorname{RCFM}_{\kappa}(R)$. But matrices in $\operatorname{RCFM}_{\kappa}(R)$ are sparse, in the sense that the entries of such matrices are few and far between. The following lemma capitalizes on this fact, giving a condition equivalent to conjugating from $\operatorname{CFM}_{\kappa}(R)$ into $\operatorname{RCFM}_{\kappa}(R)$ :

Lemma 9. Let $R$ be a right Noetherian ring, let $\kappa$ be a cardinal, and let $F_{R}=R^{(\kappa)}$. Fix $T \in \operatorname{End}\left(F_{R}\right)$. Then the following are equivalent:

(1) There exists a basis $B$ of $F_{R}$ so that $T_{B} \in \operatorname{RCFM}_{\kappa}(R)$.

(2) We have a decomposition $F_{R}=\bigoplus_{i \in I} A_{i}$ with each $A_{i}$ countably generated, free, and T-invariant.

Proof. If $\kappa \leqslant \aleph_{0}$, then condition (2) is trivial, and condition (1) holds for free by Theorem 2 So we may assume $\kappa>\aleph_{0}$. 
$(1) \Longrightarrow(2)$ : First, note if we can show condition (2) holds for a conjugate of $T$, then it holds for $T$. Second, under the identification $\operatorname{End}\left(F_{R}\right)=\operatorname{CFM}_{\kappa}(R)$, and by condition (1), it suffices to show condition (2) holds for an element $M \in \operatorname{RCFM}_{\kappa}(R)$.

We say two elements $k, k^{\prime} \in \kappa$ are related if $M_{\left(k, k^{\prime}\right)} \neq 0, M_{\left(k^{\prime}, k\right)} \neq 0$, or $k=k^{\prime}$. This is clearly a reflexive and symmetric relation, and we write $k \sim k^{\prime}$. We let $\simeq$ be the transitive closure of this relation.

We will prove that each equivalence class under $\simeq$ is countable. In fact, since $M$ is row and column finite, each $k \in \kappa$ is related to only finitely many other elements of $\kappa$. But $k \simeq k^{\prime}$ if and only if there is some $n \in \mathbb{Z}_{+}$and some elements $k_{1}, \ldots, k_{n}$ so that $k \sim k_{1} \sim \cdots \sim k_{n} \sim k^{\prime}$. For any fixed $n$, the set of all such elements is finite. So after taking the union, we have at most countably many elements.

Write $[k]$ for the equivalence class of $k$. Let $C=\left\{e_{k}\right\}_{k \in \kappa}$ be the standard basis for $F_{R}$. For each subset $\lambda \subseteq \kappa$, we write $F(\lambda)$ for the submodule $\bigoplus_{i \in \lambda} e_{i} R$ of $F_{R}$. Then one sees that $\bigoplus_{[k]} F([k])$ is the needed $M$-invariant decomposition.

$(2) \Longrightarrow(1)$ : Let $T \in \operatorname{End}\left(F_{R}\right)$. Condition (2) amounts to the fact that there is a basis $B$ of $F_{R}$ under which the matrix $T_{B}$ is block diagonal, with the blocks of countable size. By Theorem 2, we know that we can conjugate countable size matrices into $\operatorname{RCFM}(R)$, and hence we can conjugate such a block matrix also (by just conjugating each block, one at a time).

This lemma allows us to construct examples of those elements in $\mathrm{CFM}_{\kappa}(R)$ that cannot be conjugated into $\operatorname{RCFM}_{\kappa}(R)$ when $\kappa>\aleph_{0}$.

Example 10. Let $R$ be a field. To show that there is a matrix which cannot be conjugated into $\operatorname{RCFM}_{\kappa}(R)$ it suffices, due to Lemma 9, to find an uncountably generated $R[x]$-module which doesn't decompose into a direct sum of countably generated $R[x]$-modules.

Take $A_{R[x]}=R \llbracket x \rrbracket$, the power series ring over $R$. Clearly $A_{R}$ is uncountably generated and free. Suppose by way of contradiction that $A_{R[x]}=\bigoplus_{i \in I} A_{i}$, where each $A_{i}$ is a countably generated $R[x]$-module. Notice that $1 \in R \llbracket x \rrbracket$ must be contained in $\bigoplus_{j \in J} A_{j}$ for some finite set $J$. So after regrouping if necessary we may assume $1 \in A_{i_{0}}$ (for some $i_{0} \in I$ ), and hence $R[x] \subseteq A_{i_{0}}$. We have an $R[x]$ module isomorphism

$$
0 \neq \bigoplus_{i \in I \backslash\left\{i_{0}\right\}} A_{i} \cong A / A_{i_{0}}
$$

This is impossible because each element of $A / R[x]$ (treated as an $R[x]$-module) is divisible by all positive powers of $x$. But no element of $A$, besides 0 , has this property. Therefore, $A$ cannot be decomposed into the needed direct sum.

\section{ACKNOWLEDGMENTS}

I'd like to thank Victor Camillo for introducing me to this problem, the algebra group at the University of California, Berkeley for their comments on earlier manuscripts, and the referee for helpful comments.

\section{REFERENCES}

1. P. Ara, K. C. O'Meara, and F. Perera, Gromov translation algebras over discrete trees are exchange rings, Trans. Amer. Math. Soc. 356 (2004), no. 5, 2067-2079 (electronic). MR2031053 (2004k:16019) 
2. Victor Camillo, F. J. Costa-Cano, and J. J. Simón, Relating properties of a ring and its ring of row and column finite matrices, J. Algebra 244 (2001), no. 2, 435-449. MR 1859035 (2002i:16039)

3. K. R. Goodearl, P. Menal, and J. Moncasi, Free and residually Artinian regular rings, J. Algebra 156 (1993), no. 2, 407-432. MR1216477 (94f:16025)

4. J. Haefner, A. del Río, and J. J. Simón, Isomorphisms of row and column finite matrix rings, Proc. Amer. Math. Soc. 125 (1997), no. 6, 1651-1658. MR1389521 (97g:16009)

5. John Hannah and K. C. O'Meara, A new measure of growth for countable-dimensional algebras. I, Trans. Amer. Math. Soc. 347 (1995), no. 1, 111-136. MR.1282887 (95h:16027)

6. W. K. Nicholson and K. Varadarajan, Countable linear transformations are clean, Proc. Amer. Math. Soc. 126 (1998), no. 1, 61-64. MR1452816 (98d:16043)

7. K. C. O'Meara, The exchange property for row and column-finite matrix rings, J. Algebra 268 (2003), no. 2, 744-749. MR2009331 (2004i:16040)

Department of Mathematics, University of California, Berkeley, California 94720

E-mail address: pace_nielsen@hotmail.com 